\title{
A Consistent Model of Terrestrial Planet Magnetospheres and Rotations in Our Solar System
}

\author{
Fred J. Cadieu \\ Physics Department, Queens College of CUNY, Flushing, NY, USA \\ Email: fred.cadieu@qc.cuny.edu
}

How to cite this paper: Cadieu, F.J. (2021) A Consistent Model of Terrestrial Planet Magnetospheres and Rotations in Our Solar System. World Journal of Condensed Matter Physics, 11, 13-27.

https://doi.org/10.4236/wjcmp.2021.112002

Received: March 31, 2021

Accepted: May 28, 2021

Published: May 31, 2021

Copyright ( 2021 by author(s) and Scientific Research Publishing Inc. This work is licensed under the Creative Commons Attribution International License (CC BY 4.0).

http://creativecommons.org/licenses/by/4.0/

\begin{abstract}
The Sun comprises $99.9 \%$ of the solar system mass so it is expected that Sun terrestrial planet interactions can influence the motion as well as the rotation of the terrestrial planets. Gravity affects the planet orbital motions while the changing magnetic fields of the Sun can influence the planet rotations. Planets that manifest a magnetic field dominate any weaker magnetic fields from the Sun, but the rotation of terrestrial planets without a magnetic field interacts with the changing Sun's field dependent on the electrical conductivity of the core region. It is determined that the average planet density becomes a useful quantity to describe the magnetic state of a terrestrial planet. An average density of $5350 \pm 50 \mathrm{~kg} / \mathrm{m}^{3}$ is hypothesized to separate planets that develop magnetospheres from those that do not. Planets with higher average densities, Mercury and Earth, developed magnetospheres. While those with lower average densities, Venus and Mars never developed magnetospheres. Terrestrial planets with magnetospheres are the ones to also exhibit plate tectonics. The small size of Mercury led to Mercury only exhibiting a frozen in magnetization of potentially magnetic regions. The lack of magnetospheres as well as lack of plate tectonics prevented the continual transfer of core heat to the surface that limited the surface vulcanism to an initial phase. For Venus, it meant that the surface regions would only sporadically convulse. In this picture, the apparent anomalous axial rotation of Venus is a natural consequence of the rotation of the Sun. For Mars with relatively low surface temperatures, it meant that there was little heat exchange through the crust that would allow the lower crust to retain large amounts of water. For Mars to have initially had flowing liquid water required that the atmosphere at that time contained high concentrations of infrared absorbing gases at least as compared to the present level of infrared absorbing gases on the Earth. The terrestrial planets have iron based cores because iron has the highest binding energy per nucle-
\end{abstract}


on that can be made in the steady state lives of massive stars no matter how massive. This suggests that many of the conclusions reached here may also be applicable to exoplanets.

\section{Keywords}

Earth, Mercury, Venus, Mars, Magnetic Fields Sun, Early Solar System, Plate Tectonics

\section{Introduction}

In a recent publication, it was shown that the Earth acquired an anomalously high average density as a result of a collision between a pre-Earth and another object that was near the orbit of the pre-Earth [1]. This collision was extremely violent in that the object that became the Earth wound up with a major portion of the more massive and also radioactive elements. An indication of this is that the observed isotopic ratios of the Earth and what then became the moon should be the same [2]. As the debris field moved out beyond the Roche limit, the moon formed as an elongated mass so that it orbited the Earth with the same side facing the Earth as it gradually migrates out away from the Earth. From the aftermath of this collision, the Earth Moon system was the result. In that previous publication, some results from the Earth Moon formation were discussed. A principal result was that the Earth acquired an anomalously high average density that led to Earth exhibiting a sizeable magnetosphere. This magnetic field allowed the development of plate tectonics making possible long-term billion-year stability. Such long-term stability is generally recognized as being necessary for the development of complex life forms. In this publication, some consequences and possible generalizations to other terrestrial planets will be discussed. Since Earth has a magnetic field, the general tendency is to assume, that other planets started out with a magnetic field that was then subsequently lost due to quirks of development. The objective of this paper is to show that there is a pivotal link between the details of the Sun to planet interaction, the rotation of the planets, and whether a terrestrial planet manifests a magnetosphere. It should be noted that the Sun comprises $99.9 \%$ of the entire solar system mass so that Sun planet interactions can be expected to be important. The Sun interacts with the planets mainly through gravity, but the Sun also interacts with the various planets through the Sun's changing magnetic fields. The Sun has both a dipolar magnetic field that is currently changing polarities about every 11 years and radial components associated with solar wind and the Parker spiral. The interaction with the comparatively weak fields from the Sun depends upon whether the planet has a magnetic field of its own and the electrical conductivity of any core regions. This is because Lenz's law states that currents in a conductor will always be generated to oppose any magnetic flux changes. 
A key goal of this paper is to examine some of the reasons that a terrestrial planet exhibits a magnetic field. The terrestrial planets have iron based cores since iron has the highest binding energy that can be made in the steady statelives of massive stars. Iron is then especially abundant in the cores of terrestrial planets far and wide. Iron is expected to be a widely common element in the cosmos. Our solar system is just one example of a solar system formed under the general nebular theory of solar system formation. [3] [4] This suggests that the conclusions reached here are also applicable to exoplanets currently being discovered.

\section{Results and Discussion}

For a terrestrial planet to exhibit a magnetic field there is a general understanding that there must be a molten outer core region and sufficient rotation so that currents can through convective action generate a magnetic field. For terrestrial planets the major core elements are iron and some combination of nickel and other metallic elements. Iron acquired its universal abundance because it is the most massive element that can be made in the steady state lives of the cores of massive stars no matter how massive. Iron is generally regarded as a ferromagnetic element, but for the purpose of generating a magnetic field for the earth the key thing is that it can act as an electrical conductor. Similarly currents in a coil of copper wires can generate a magnetic field even though copper itself is not a magnetic element. A goal of this paper is to parameterize the factors that lead to a consistent picture of the magnetospheres of the four terrestrial planets and the moon in the solar system. It should be noted that our solar system has what may be termed a friendly Jupiter so that the general placement and development of the terrestrial planets has not been seriously altered since the formation of the solar system. The key driver for the development and evolution of the terrestrial planets was and is the upwelling of heat from interior regions. The core region of a terrestrial planet comprises only a small fraction of its total mass. For the Earth, for example, it will be shown that the mass fraction within a sphere one-fourth of the Earth radius is just 2\% of the Earth's total mass. But the upwelling of heat from that small fraction of the total mass is extremely crucial for the Earth's magnetosphere. Table 1 contains some useful relevant data for the terrestrial planets as a subset of NASA data tables [5].

The terrestrial planets in our solar system formed in the presence of a thermal gradient with higher temperatures occurring nearer the Sun. The Sun and the planets had a common formation time of 4.6 billion years ago. As stated earlier the Sun comprises $99.9 \%$ of the mass in the solar system. As shown in Ref. 1 the average density of the terrestrial planets Mercury, Venus, and Mars follow a smooth curve with the Earth Moon system being anomalous because of the Earth formation process. Useful parameters for characterizing the evolution of a terrestrial planet might include the total mass, the mass and radioactive element concentration as a heat source, the average planet density, and some measure of 
Table 1. NASA terrestrial planet details. Only a subset of listings are included here [3].

\begin{tabular}{cccccccc}
\hline Planet & $\begin{array}{c}\text { Mass } \\
(\text { Earth }=1)\end{array}$ & $\begin{array}{c}\text { Semimajor } \\
\text { Orbit } \\
\text { Radius } \\
\text { (AU) }\end{array}$ & Eccentricity & $\begin{array}{c}\text { Orbit } \\
\text { Period } \\
\text { (Solar } \\
\text { Days) }\end{array}$ & $\begin{array}{c}\text { Rotation } \\
\text { Period } \\
\text { (Solar } \\
\text { Days) }\end{array}$ & $\begin{array}{c}\text { Axial } \\
\text { Tilt } \\
\text { Angle }\left(^{\circ}\right)\end{array}$ & $\begin{array}{c}\text { Average } \\
\text { Density }\end{array}$ \\
(Earth $=1)$
\end{tabular}

the planet electrical conductivity of a core region as a means of interacting with the Sun through the Sun's magnetic field. Terrestrial planets are expected to exhibit gravitational differentiation so that more dense regions sink to the interior regions. Figure 1 shows the average density of the terrestrial planets as shown in Ref. 1. The Earth acquired an anomalously high density as a result of the Earth-Moon formation process as also shown in Figure 1.

Fairly recent publications have presented measurements and modeling of the density of the Earth as a function of the depth into the Earth [6] [7]. These measurements can be recast to represent density as a function of the radius of the Earth by subtracting the depth from the Earth average radius $=6371 \mathrm{~km}$. Figure 2 shows the density of the Earth as a function of the radius from the center. There is a density drop in moving outward from the inner core to the outer core. This is interpreted as a transition from a solid inner core to a fluid outer core. There is also a fairly rapid decrease in moving outward from the outer core to the lower mantle.

Table 2 shows the integral of the density for the different regions of the Earth. Even though the core density rises to about 13.1 times the density of water the total mass fraction within this region is only about 0.017 of the total mass. This occurs for two reasons. One is that even in the outer most regions the density is still 3 to 4 times the density of water. And reason two is that the volume of a sphere is a steep function of radius being proportional to radius cubed. Integrating out to the Earth's surface gives the total Earth mass of $5.97 \times 10^{24} \mathrm{~kg}$. 


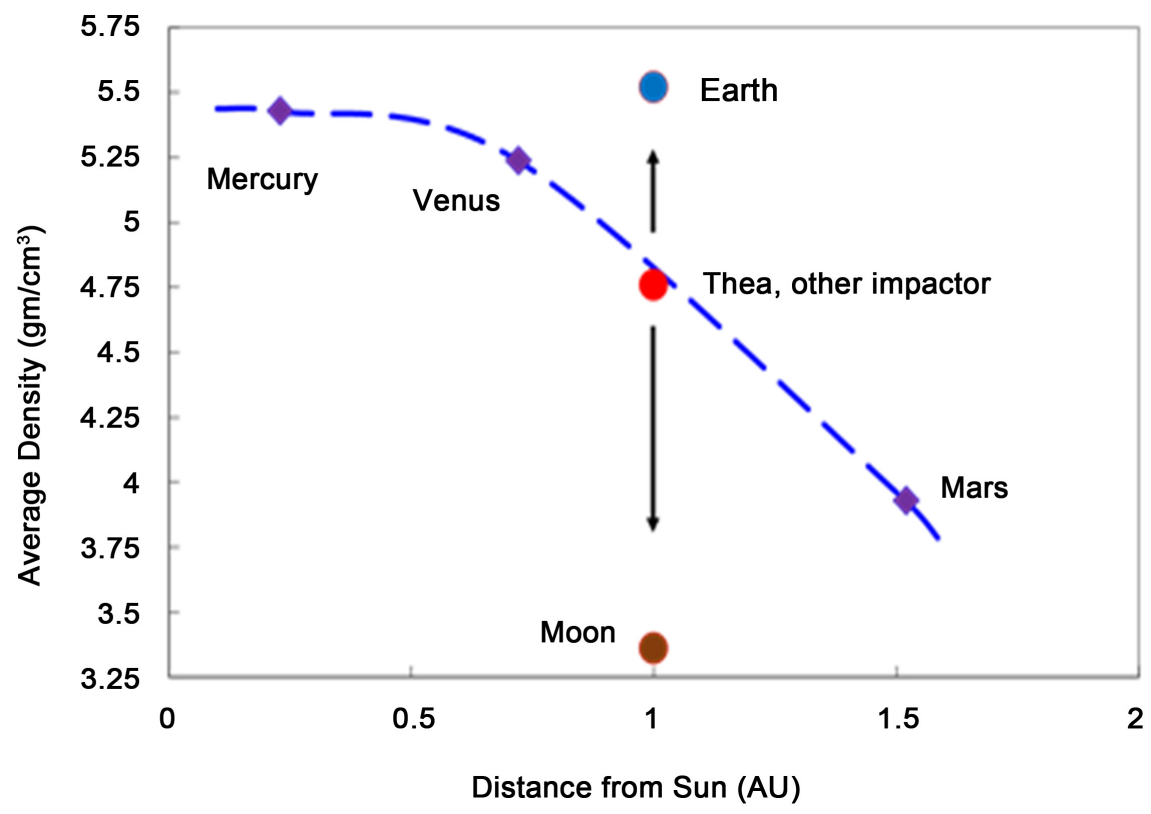

Figure 1. The dotted line shows the variation in the average terrestrial planet density as a function of the distance from the Sun [1]. The anomalous earth and moon average densities are also indicated.

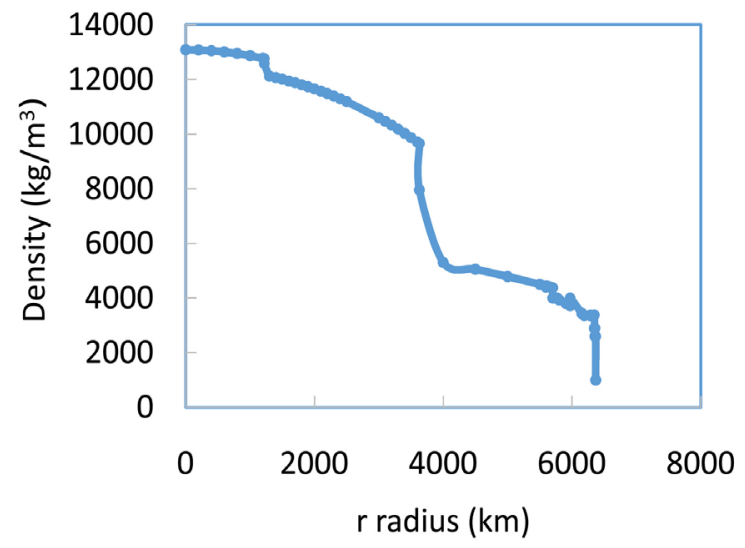

Figure 2. The density of the Earth as function of radius is shown as determined from the data in [4] and [5].

Dividing the total mass by the Earth volume gives an average Earth density = $5515 \mathrm{~kg} / \mathrm{m}^{3}$. It is interesting to note that Earth density as a function of $\mathrm{r}$ does not fall to this low a level until $r \approx 0.63 \mathrm{R}_{\mathrm{E}}$.

For Earth, planet rotation, and rising heat convection produces currents in the fluid outer core that produces a magnetic field. This continuous rising heat associated with the magnetic field is what drives plate tectonics on Earth. The Earth has sufficient size so that the supply of heat provided by the original compaction energy plus that from radioactive decays is expected to last the foreseeable time scale as set by the stability of the Sun [8]. The half-life for the radioactive decay of $\mathrm{U}^{238}$ is 4.5 Billion years so half of the original $\mathrm{U}^{238}$ still remains now.

Each of the terrestrial planets as well as the moon has a core region composed 
Table 2. Earth regions densities and mass fractions. $\mathrm{R}$ Earth $=6371 \mathrm{~km}$. Mass of Earth $=$ $5.97 \times 10^{24} \mathrm{~kg}$. Earth regions have been chosen to facilitate integration of the density as a function of radius.

\begin{tabular}{|c|c|c|c|c|}
\hline Region & Radius (km) & Density $\left(\mathrm{kg} / \mathrm{m}^{3}\right)$ & $\begin{array}{c}\text { Integrated } \\
\text { Mass } \\
\left(10^{24} \mathrm{~kg}\right)\end{array}$ & $\begin{array}{c}\text { Mass } \\
\text { Fraction \% }\end{array}$ \\
\hline Inner Core & $0-1221.5$ & $\begin{array}{c}13,088.5 \\
-2.1750 \times 10^{-7} \times \mathrm{r}^{2}\end{array}$ & 0.09992 & 1.674 \\
\hline Outer Core & $1221.5-3480.0$ & $\begin{array}{c}12,500.0 \\
-5.347 \times 10^{-4} \times(\mathrm{r}-1221.5)^{2}\end{array}$ & 1.887 & 31.608 \\
\hline Lower Mantle & $3480.0-3600.0$ & 7956.5 & 0.1504 & 2.5193 \\
\hline Mantle & $3600.0-4000.0$ & $\begin{array}{c}7956.5 \\
-6.57 \times(\mathrm{r}-3600)\end{array}$ & 0.482 & 8.074 \\
\hline Mantle & $4000.0-6200.0$ & $\begin{array}{c}5200 \\
-4.1729 \times 10^{-4} \times(\mathrm{r}-4000)^{2}\end{array}$ & 3.1996 & 53.590 \\
\hline \multirow[t]{2}{*}{ Crust } & $6200-6371$ & $\begin{array}{c}2690 \\
-9.766 \times(\mathrm{r}-6200)\end{array}$ & 0.1568 & 2.626 \\
\hline & & & $\begin{array}{c}\text { Total mass }= \\
5.975\end{array}$ & 100.09 \\
\hline
\end{tabular}

largely of iron, but the core regions differ in their temperatures and pressures. Consequently, the cores differ in the phases from partially solid and fluid with the denser solid core being surrounded by a lower density fluid. Lower pressures due to either lower mass compacting the planet or lower temperatures can produce either fluid cores or solidified cores. The phase diagram of iron has been measured so that the Earth core temperature and pressure profiles are well established [9]. The Earth's magnetic field is definitely associated with the core region. The Earth's magnetic field poles have been measured to flip on the order of half a million of years indicating the Earth's core must be a main factor to have such a long period [10]. The mantle contains nearly $2 / 3$ rds of the total mass as shown in Table 2. The composition of the mantle for each of the terrestrial planets and the moon should be largely similar.

Before considering the details of any particular terrestrial planet it is useful to consider some of their common formation elements. Earth experienced a period known as the Hadean Eon from formation time to about 700 million years [11]. In this time before the start of life similar developments should have happened on the other terrestrial planets. Each of the terrestrial planets formed as a part of the formation of the Sun. Each acted to compact the mass at some distance from the Sun with sufficient heat to cause gravitational differentiation. Each then experienced a period of volcanoes causing surface disruptions. Volatile constituents such as water were largely boiled away from the surfaces of the forming planets. It is uncertain what fraction of water was returned via comets versus from outgassing from deeper within the Earth. The fraction returned via comets is generally held to be a sizable amount from $10 \%$ to $100 \%$. Evidence is that the surface of the Earth was entirely covered by liquid water early in its history [10]. 
This was probably true for at least the planets Venus, Earth and Mars.

Each terrestrial planet shared a time period of rapid bombardment early in the history of the solar system. Each body was intercepted by a great number of water bearing comets in proportion to the cross section of the planet. The cross section, $\pi R^{2}$, and the surface area, $4 \pi R^{2}$, are both proportional to the radius squared so there is the initial expectation that the ocean depth contribution from comet impacts on the different planets would initially be the same. The time scale for this initial bombardment and acclimation phase is expected to be certainly less than a billion years. Each of the planets whether from initial planet outgassing, or from comet bombardment, was initially covered in water. Each of the planets then lost or retained an initial water covering at a rate dependent on the planet distance from the Sun and energy evolution. For Earth rudimentary life is generally thought to arise by about a billion years. Since no evidence of life on any other terrestrial planet has been observed, processes that cause the differentiation of development on the different terrestrial planets had become effective on the different planets. This is probably a period long enough for geologic relaxation, but not long enough for the development of any but the earliest forms of life. Early in the Sun's history the luminosity was only about $70 \%$ of the current value [1]. To explain how the Earth could have been warm enough to have liquid flowing water two items are generally thought to have been present. One is that there was enough water present to have largely covered the Earth's surface [12]. Water reflects less sunlight than land areas so that the lowered albedo causes greater heat absorption. The second is that there is expected to be $\mathrm{CO}_{2}$ outgassing associated with vulcanism and surface disruptions [13]. Each of the terrestrial planets would have experienced a greater amount of heating due to water covering most of the surfaces initially. Mars, in particular, should have had an initial period of water coverage and large surface disruptions. Even Mercury and Venus would have had an initial period of water coverage and surface changes. For Earth this period of initial planet development is called the Hadean Eon and lasted about 0.7 billion years [11]. How long such a period lasted on the other terrestrial planets is much less certain and starts to depend on planet details.

Mars shows evidence of flowing water early in its history. An examination of the conditions for Mars to have surface temperatures high enough for flowing liquid water can be obtained by examination of the energy balance coming from the Sun and that radiated by Mars. Early on the Sun had a luminosity only about $70 \%$ of its current value [1]. The basic condition for a planet to have a covering of liquid water is that the planet surface temperature be greater than $273 \mathrm{~K}$.

The equation below simply states that the energy received by a planet must equal the radiated energy for the planet to maintain the temperature $T$.

$$
x \cdot L \cdot \frac{\pi R_{p}^{2}}{4 \pi D_{S-p}^{2}} \cdot(1-a) \cdot(1+\tau)=4 \pi R_{p}^{2} \cdot \sigma \cdot T_{p}^{4}
$$

$L=$ present Luminosity of the Sun, $x \cdot L=$ fraction of present Sun's Luminosity. 
$R_{p}=$ planet radius, $D_{S_{-p}}=$ distance Sun to planet.

$a=$ planet albedo equal to fraction of incoming energy reflected back to space, $\tau=$ number of times infrared wavelengths are absorbed in traversing the planet's atmosphere, $\sigma=$ Stefan-Boltzmann constant, $T_{p}=$ planet surface temperature.

For Figure $3 x \cdot L$ has been chosen to be $70 \%$ of the Sun's luminosity to represent the luminosity early in the lifetime of the Sun. The factor a has been chosen to be 0.30 which is close to the present albedo of the Earth. An albedo of 0.3 can result from either some clouds or some partial reflective covering from snow or ice. It is probably a reasonable value. At the present time, $x=1$, for the Earth using $a=0.30$ and $\tau=1.00$ gives the average surface temperature for the Earth. At the present time for Venus using $a=0.70$ and $\tau=70$ gives the average surface temperature for the planet Venus. The quantity $\tau$ is often called the "absorption length" even though it does not have the dimensions of a truelength. The principal infrared absorbing, greenhouse, gases are water vapor, carbon dioxide, and methane. Diatomic gasses such as $\mathrm{N}_{2}$ and $\mathrm{O}_{2}$ do not appreciably contribute to Tau since they are not strong infrared absorbers. All of the different gases contribute to thermal transfer processes as expressed by the ideal gas law.

These calculations indicate that for Mars to have had liquid water on its surface a minimum Tau at least 4 was required. Since at the present time for Earth, $x=1$, water vapor plus about $280 \mathrm{ppm} \mathrm{CO} \mathrm{CO}_{2}$ gives Tau $\approx 1$, then $\mathrm{CO}_{2}$ levels must have been about 4 times current Earth values to have had liquid water flowing on Mars early in its history. This is the case that Mars would have liquid water but at temperatures barely above the freezing point of water. The vapor pressure of water as a function of temperature is very nonlinear [1] so that surface evaporation would have been slow. Solar wind emanating from the Sun is generally credited with slowly stripping away surface water and most of the atmosphere of

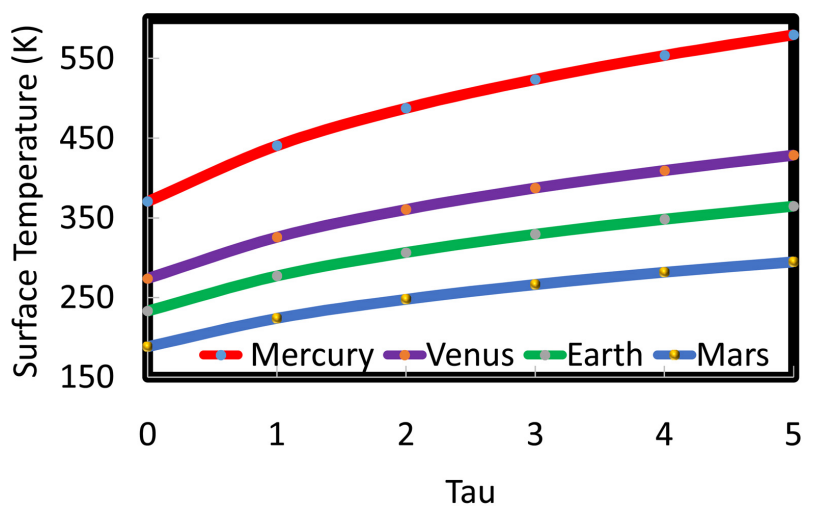

Figure 3. Early Faint Sun at 70\% of current solar luminosity energy balance surface planet temperatures. An albedo of 0.30 has been used for these calculations. Conditions of different planetary atmospheres have been indicated due to varying absorption lengths. For each planet surface temperatures have been computed for different values of Tau. Water vapor and atmospheric $\mathrm{CO}_{2}$ are generally the major contributors to Tau. 
Mars [14]. Due to the relatively small size of Mars as vulcanism seized there was little source of heat to promote further outgassing of water to the surface. Since Mars does not exhibit plate tectonics there was not a regular transfer of heat from the interior regions to the surface. Sporadic large scale vulcanism has been determined to have extended up to about 150 million years ago [15]. The timescale of Martian vulcanism is very long so that even some future events may be expected. It is therefore expected that Mars could have retained large amounts of water below the surface in crustal regions [16].

The energy balance equation in the form presented is not generally applicable to the planet Mercury, nor to the Moon, since these bodies do not have atmospheres. But early on during initial formation there would be a period of initial outgassing and vulcanism in which Mercury would have had an initial atmosphere. The high temperatures indicated for Mercury in Figure 3 indicate that there would not have been a time of quiet oceans on Mercury but that a boiling state would naturally tend to occur.

Venus is indicated to have been relatively warm early on so that there would have been a rapid transfer of water to the upper atmosphere where it would have been rapidly dissociated by ultraviolet light from the Sun leading to $\mathrm{H}$ escaping to space. Over a relatively short time $\mathrm{H}$ and hence water would have been lost to space by this mechanism. The Venusian atmosphere would have then become more and more enriched in carbon dioxide as vulcanism continued. Earth would have had a much lower rate of water transport to the upper atmosphere since the vapor pressure curve for water is highly nonlinear [1]. Consequently, Earth still had a significant level of water even up to the time in which oxygen became a significant component of the atmosphere, so that ozone in the upper atmosphere could limit the escape of $\mathrm{H}$ to space. Oxygen became a significant part of Earth's atmosphere only after about two billion years [17].

An interesting question then is how the Earth would have to be changed so that it would no longer exhibit a stable long term magnetic field. Any change that eliminates the change in density between the inner core and outer core would remove the conditions favoring a magnetic field. Since the inner core comprises only $1.7 \%$ of the Earth's total mass the transition to a nonmagnetic Earth can be facilitated with very little change in the total mass. In this regard the average density then becomes a very good indicator of the planets' behavior. Earth has the highest average density of the terrestrial planets. It was the basic conclusion of a previous paper that this was the result of the collision that resulted in the Earth-Moon system [1]. Venus has a mass slightly less and a lower average density. Venus has no magnetosphere. It is postulated that an average density greater than about $5350 \pm 50 \mathrm{~kg} / \mathrm{m}^{3}$ is required for the maintenance of a long-term magnetic field. On this basis Earth and Mercury should have started out with magnetospheres and magnetic fields. There is the expectation that Earth with its anomalously high average density and large size retained the magnetic field. The small size of Mercury facilitated core changes over time that it is expected that Mercury would presently exhibit a fossilized or frozen out 
magnetic field. Venus and Mars, as well as the Moon, then never developed stable magnetospheres or magnetic fields.

The Sun exhibits a dipolar magnetic field associated with convection currents in the metallic hydrogen of the convection zone. Presently the dipolar field has been reversing polarity about every 11 years. At the present time the equatorial region of the Sun has a rotation period of about 24 days with a longer period as the field becomes more concentrated nearer the poles up to about 34 days. This is an example of magnetic breaking in action. Extending outward away from the Sun the Sun's magnetic field aided in transferring angular momentum away from the Sun to the forming planetary system. The general rotation and expected rotation of the Sun and of the various planets is illustrated in Figure 4. Viewed from above if the Sun is rotating anticlockwise then it is expected that all the planets of the solar system would also be orbiting anticlockwise. The rotation period of the Sun, currently about 24 days, is much less than the orbital rotation period of any of the planets. The daily axial rotation of the Earth is also in the expected direction as illustrated in Figure 4. The Earth has a magnetosphere that largely isolates the Earth from the weaker magnetic fields from the Sun. The dominance of the Earth's magnetic field is indicated by the dotted circle surrounding the Earth in Figure 4. The most dramatic evidence for the action of the Earth's magnetic field is the aurora borealis due to charged particles emanating from the Sun moving toward the North and South poles of the Earth. The solar wind emanating from the Sun follow axial magnetic field lines away the

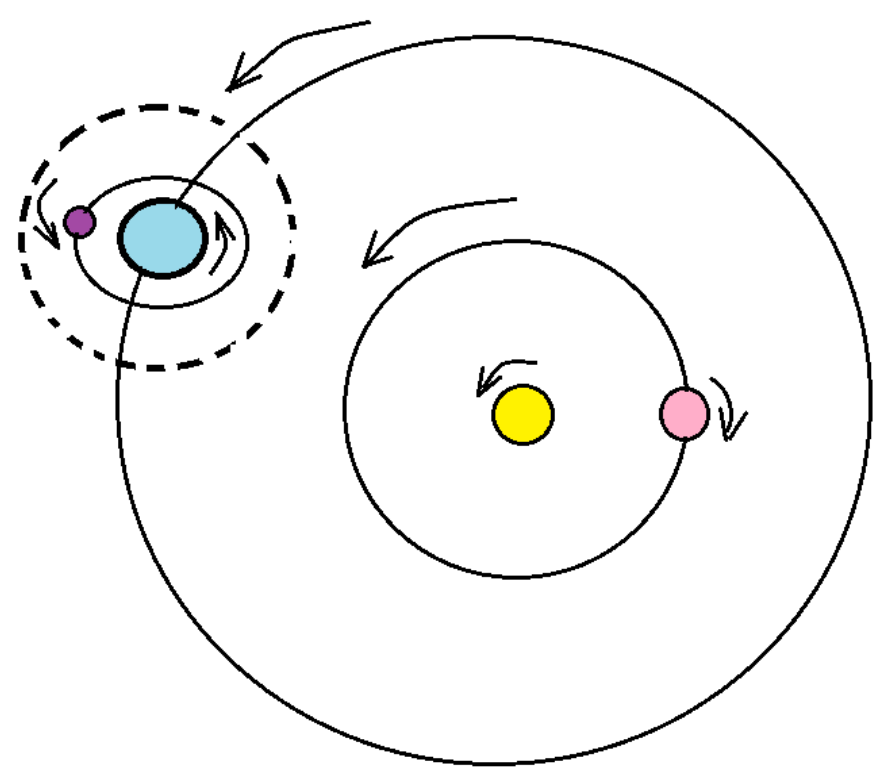

Figure 4. The orbital rotation of the planets, Earth and Venus, and the Sun are indicated. The rotation period of the Sun is currently about 25 days, but was initially shorter. Venus interacts directly with the Suns magnetic field and rotates clockwise as it orbits the Sun in a counterclockwise direction. The Earth was shielded by its own magnetic field from the weaker rotating magnetic field from the Sun. 
Sun. The axial rotation of Mercury, although slow, and Marsare also in the expected anticlockwise direction. Venus, however, has an axial rotation that is clockwise as indicated in Figure 4. The Sun has also a radial magnetic field associated with the solar wind.

On this basis since Venus never developed a magnetosphere the metallic conducting core was directly subject to the magnetic field of the Sun. It should also be noted that the early Sun was then rotating at a faster rate than at present that increased its interaction with Venus. Initially a relatively rapid rotating Sun emitted sufficient solar wind to blow outward the initial primordial hydrogen rich atmosphere from the inner solar system [18]. If the Sun was still rotating too rapidly indications are that planets such as the Earth would not have been able to develop stable secondary atmospheres. It is argued that life was only able to develop on the Earth because of the Goldilocks rotation rate of the Sun. [18] The Sun has both a dipolar time varying magnetic field as well as a spirally outward magnetic field that act to slow the rotation of Venus as well as dragging Venus as a poorly coupled gear. The dipolar field of the Sun presently reverses about every 11 years and may have been a shorter time in the past as the Sun rotation rate is also thought to have been faster. Lenz's law would then say that eddy currents would be generated in Venus to magnetically brake the rotation of Venus. The current directions for the eddy current loops indicated in Figure 5 would be in opposite directions and the directions would reverse as the polarities

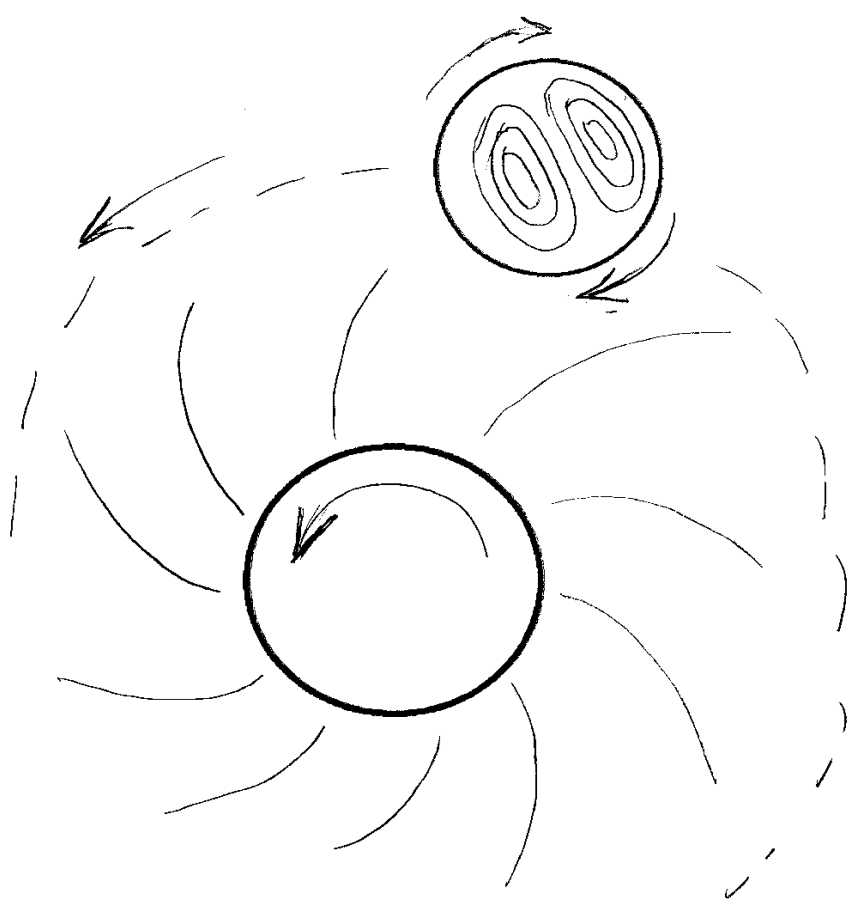

Figure 5. The relatively rapid rotation of the Sun and the nearly redial magnetic field associated with the solar wind are indicated. Eddy currents in Venus that switch directions with the polarity reversing dipolar field of the Sun are indicated. The axial rotation direction of Venus is set by the dragging action of the rotating Sun. 
of the Sun's dipolar field reverses. This is shown in Figure 5. The results shown here are only qualitative. Further work toward quantitative solutions is planned. As indicated in Table 1 there is a very small angle between the rotation axis of both Mercury and Venus and the Sun. These planets are then ideally oriented to interact with the rotation of the Sun. The radial component of the relatively rapid rotating Sun drags the closer side of Venus forward resulting in a clockwise rotation of Venus in Figure 5. It may help to visualize the Sun as a rotating gear acting to spin Venus. The clockwise rotation of Venus then naturally results from the rotation of the Sun. The Earth's magnetosphere shields the Earth's rotation from directly interacting with the weaker magnetic fields from the Sun. The Sun's magnetic field acted on each of the terrestrial planets dependent on the magnetosphere of the planet as well as the electrical resistivity of the core.

Mercury initially would have had a magnetosphere which shielded the magnetic core of Mercury from the Sun's weaker magnetic field. The small size of Mercury leads to a cooling and solidifying of the core so that Mercury over a certain time frame lost the ability to generate a magnetic field of its own. It should then exhibit two manifestations of this. One should be a frozen in magnetization of any magnetic deposits. Recent measurements made by the Messenger space craft indicate that Mercury exhibited a magnetic field up to about 4 billion years ago [19]. The other should be that the metallic core of Mercury would then interact with the changing magnetic fields of the Sun to brake any rapid rotations of Mercury. The planet orbits have been approximated as circular which limits any detailed analysis, particularly for Mercury, which exhibits a large eccentricity as shown in Table 1.

Further out in the solar system Mars red color indicates an incomplete differentiation. The average density of Mars at $3934 \mathrm{~kg} / \mathrm{m}^{3}$ is very low to have a highly electrically conducting core. The average density of Mars is well below that hypothesized to be required for a terrestrial planet to manifest a magnetic field. The behavior of Mars can then be reconciled with that of the other terrestrial planets by Mars having a higher core resistivity to limit the generation of eddy currents as a means of the planet interacting with the changing Sun's magnetic field. Mars has a relatively rapid axis rotation with a day currently about equal to that of the Earth. The rotation period of the Earth is increasing as the Moon gradually moves away from the Earth. Initially a day on the Earth was much shorter than that of a Mars day but at the present time each has a similar length of day [1]. Mars then exhibits a rotation rate that has largely remained unchanged since the formation of the early solar system. The axial rotation angle for Mars is relatively large at about 25 degrees as shown in Table 1 so that Mars rotation is largely decoupled from that of the Sun. Mars does presently have two small moons but the ratio of the mass of Mars to that of the moons is 53 million so that the motion or capture of the moons is not expected to change the motion of Mars appreciably. For comparison the ratio of the mass of Earth compared to Earth's moon is about 83 times. 


\section{Conclusions}

Bodies in the usniverse manifest a magnetosphere by two principal methods. The terrestrial planets are a separate group that may or not exhibit a magnetosphere dependent on the details of the iron based core. Here it has been postulated that a minimum average density of $5350 \pm 50 \mathrm{~kg} / \mathrm{m}^{3}$ is required for a terrestrial planet to exhibit a stable magnetosphere. An iron based core results from the fact that iron is the element with the maximum binding energy per nucleon that can be made in the steady state of high mass stars. On this basis Mercury would have initially exhibited a magnetic field which was frozen out as the core cooled. Earth, with a larger fraction of heavier elements and radioactive elements acquired in the highly energetic event that created the Earth-Moon system has a stable magnetosphere that is expected to last the projected lifetime of the Earth. The rotation rate of planets with highly conducting cores without a magnetic field for shielding is limited by the interaction with the changing dipolar magnetic field of the Sun. Thus Mercury, once its own magnetic field is frozen out, and Venus, are limited to slow rotations by the changing dipolar magnetic field of the Sun. Both the rotation axes of Mercury and Venus are within several degrees to that of the Sun and situated for maximum interaction. Mars on the other hand has an axial rotation axis that is tilted about $25^{\circ}$ from that of the Sun and is decoupled from the Sun. The relatively low average density of Mars indicates that the core of Mars has limited conductivity that precludes interactions with the Sun's changing magnetic fields.

The other types of magnetospheres depend on high pressure and density hydrogen. All gas giant planets as well as all stars exhibit magnetic fields which depend on convection currents that are not associated with core details or structures. A useful search would be to find a gas giant planet, or a star, that does not have a magnetic field. There does not seem to be any minimum average density required for such objects to exhibit a magnetic field; they all exhibit atomic hydrogen originated magnetic fields. The roughly half million year pole flipping period of the Earth indicates a magnetic field that is associated with the core of the Earth. While the rapid pole flipping time of the Sun of only about 11 years at present indicates that the Sun's magnetic field is not associated with the massive core of the Sun.

The energy balance equation and Figure 3 show that the distance of the terrestrial planet from the Sun plays a crucial role on the water state on the surface of the planet. The planet surface temperature must have been at least $273 \mathrm{~K}$ to have flowing liquid water. Dissolved salts may act to slightly lower this required temperature. For Mars to have had flowing liquid water, the energy balance equation requires that a relatively high concentration of infrared absorbing gases must have been present in the early Martian atmosphere. A value of at least about 4 times the current Earth value must have been present before the Mars surface dried. Drying is from the surface and for a relatively stable and cool crust a lot of water would have been retained in the crust. Mars does not exhibit plate tecton- 
ics so that there is little mixing of deeper crustal water with an original surface coating.

The terrestrial planets have iron based cores because iron has the highest binding energy per nucleon that can be made in the steady state lives of massive stars no matter how massive. The elemental abundances of different elements in the terrestrial planets are largely set by the shape of the binding energy per nucleon curve. This curve is set by fundamentally nuclear properties independent of any particular planet or star. This suggests that many of the conclusions reached here may also be applicable to exoplanets.

\section{Conflicts of Interest}

The author declares no conflicts of interest regarding the publication of this paper.

\section{References}

[1] Cadieu, F.J. (2020) Just a Bit of Physics Can Tell So Much: A Unique Story of the Star of the Earth-Moon System. World Journal of Condensed Matter Physics, 10, 88-103. https://doi.org/10.4236/wjcmp.2020.102006

[2] Dauphas, N. (2017) The Isotopic Nature of the Earth's Accreting Material through Time. Nature, 541, 521-524. https://doi.org/10.1038/nature20830

[3] Woolfson, M.M. (1993) Solar System-Its Origin and Evolution. The Quarterly Journal of the Royal Astronomical Society, 34, 1-20.

https://ui.adsabs.harvard.edu/abs/1993QJRAS..34....1W

[4] Wikipedia (n.d.) Nebular Hypothesis.

[5] National Aeronautics and Space Administration (NASA) (2021) Only a Subset of the NASA Entries Are Listed. https://solarsystem.nasa.gov/planet-compare/

[6] Dziewonski, A.M. and Anderson, D.L. (1981) Preliminary Earth Model. Physics of the Earth and Planetary Interiors, 25, 297-356. https://doi.org/10.1016/0031-9201(81)90046-7

[7] Kennett, B.L.N. (1998) On the Density Distribution within the Earth. Geophysical Journal International, 132, 374-382. https://doi.org/10.1046/j.1365-246x.1998.00451.x

[8] Johnston, H. (2011) Radioactive Decay Accounts for Half of Earth's Heat. https://physicsworld.com/a/radioactive-decay-accounts-for-half-of-earths-heat

[9] Alfe, B.D., Gillan, M.J., Vocadlo, L., Brodholt, J. and Price, G.D. (2002) The ab Initio Simulation of the Earth's Core. Philosophical Transactions of the Royal Society A, 360, 1227-1244. https://doi.org/10.1098/rsta.2002.0992

[10] Hazen, R.M. (2012) The Story of Earth. Penguin Books, New York, 117-119

[11] Hazen, R.M. (2012) The Story of Earth. Penguin Books, New York, Ch. 1-5.

[12] Johnson, B.W. and Wing, B.A. (2020) Limited Archaean Continental Emergence Reflected in an Early Archaean ${ }^{18} \mathrm{O}$-Enriched Ocean. Nature Geosciences, 13, 243-248. https://doi.org/10.1038/s41561-020-0538-9

[13] Thompson, A. (2010) Clouds May Hold Key to Why Early Earth Didn't Freeze Over. http://space.com

[14] Jakosky, B. (2017) Solar Wind Stripped Martian Atmosphere Away. 
https://www.sciencedaily.com/releases/2017/03/170330142211.htm

[15] Martel, L.M.V. (2011) VulcaTimeline of MartianVulcanism, Planetary Science Research Discoveries. http://www.psrd.hawaii.edu/May11/Mars volc timeline.html

[16] Scheller, F.I., Ehlmaan, B.L., Hu, R., Adams, D.J. and Yung, Y.L. (2021) Long-Term Drying of Mars by Sequestration of Ocean-Scale Volumes of Water in the Crust. Science, 372, 56-62. https://doi.org/10.1126/science.abc7717

[17] Hazen, R.M. (2012) The Story of Earth. Penguin Books. New York, Ch. 7.

[18] Dorminey, B. (2018) Early Sun's 'Goldilocks' Rotation Rate May Be Why We're Here.

[19] Johnson, C.L., Phillips, R.J., Purucker, M.E., Anderson, B.J., Byrne, P.K. and Denevi, B.W. (2015) Low-Altitude Magnetic Field Measurements by Messenger Reveal Mercury's Ancient Crustal Field. Science, 348, 892-895.

https://doi.org/10.1126/science.aaa8720 